\title{
Editorial: Diacylglycerol Kinase Signalling
}

\author{
Isabel Merida ${ }^{1 *}$, Andrea Graziani ${ }^{2,3}$ and Fumio Sakane ${ }^{4}$ \\ ${ }^{1}$ Department of Immunology and Oncology, CNB-CSIC, Madrid, Spain, ${ }^{2}$ Department of Translational Medicine, University of \\ Piemonte Orientale, Novara, Italy, ${ }^{3}$ Division of Experimental Oncology, School of Medicine, Vita-Salute San Raffaele \\ University, Milan, Italy, ${ }^{4}$ Department of Chemistry, Graduate School of Science, Chiba University, Chiba, Japan
}

Keywords: lipid signaling, immune system, synaptic transmission, immunotherapy of cancer, cytotoxic $t$ cells, synaptic plasticity (LTP/LTD)

\section{Editorial on the Research Topic}

\section{Diacylglycerol Kinase Signalling}

By gathering 10 reviews from leading scientists in a fast-evolving field, this Research Topic illustrates the contribution of diacylglycerol kinase (DGK) family members to the regulation of cell responses, and how their malfunction contributes to human pathologies.

DGK transform diacylglycerol (DG) into phosphatidic acid (PA). This simple reaction alters the levels of two lipids with central functions as second messengers, as phospholipid precursors, and as membrane modulators. The first DGK was purified and cloned by the Hideo Kanoh group in 1990; since then, the study of DGK regulation and functions has expanded and grows daily. Mammalian DGK comprise 10 isoforms that are particularly abundant in brain and hematopoietic organs. Not surprisingly, DAG effectors such as protein kinase C family members, mammalian Unc13 homologs, the chimaerin family of Rac GTPases, and the Ras GEF RasGRPs have critical functions in the regulation of nervous and immune synapses.

The mammalian DGK isoforms are grouped into five subtypes based on the presence of distinct regulatory domains. At least eight DGK isoforms are detected in the mammalian brain. In their review, Lee et al. summarize studies that link various DGK isoforms with certain features of synaptic plasticity. The generation of knockout mice for specific DGK isoforms has demonstrated their involvement in distinct aspects of brain function. These findings imply precise, isoformspecific control of lipid homeostasis that creates local environments suitable for triggering synaptic plasticity. Genome-wide association studies suggest relationships between specific DGK and human diseases that include bipolar disease and Parkinson's disease. Sakane et al. review these findings and discuss others such as mutations in the DGKE gene and its link to epilepsy and Huntington disease, or the more recent discovery of DGKE mutations associated with atypical hemolytic-uremic syndrome (aHUS).

The predominance of DGK $\varepsilon$ malfunction and disease might be related to this isoform's unique properties. DGK $\varepsilon$ is the only DGK family member that lacks regulatory domains, that is membranebound, and has substrate specificity for arachidonate-containing DG. Two articles in this series review DGK $\varepsilon$ properties and functions in depth, and cast new light on the links between DGK $\varepsilon$ defects and disease. Epand et al. describe the structural features that lend DGK $\varepsilon$ its arachidonoylDG specificity, its regulation by phosphorylation, and the identification of DGK $\varepsilon$-interacting proteins. They discuss at length the unanticipated connection between recessive DGKE mutations and aHUS, recently uncovered by whole-exome sequencing. Although dysregulated complement activation is generally associated with all aHUS forms, the vast majority of aHUS patients that harbor DGKE mutations do not show this defect. DGK $\varepsilon$ is found at the endoplasmic reticulum (ER), a specialized site in membrane phospholipid synthesis and transport. In their review, Nakano et al. discuss the $\mathrm{DGK} \varepsilon$-specific contribution at the ER by generating arachidonoyl PA that 
feeds directly into the PI cycle. DGK $\varepsilon$ malfunction impairs PA generation and facilitates DG lipase-dependent DG degradation to 2-arachidonoyl glycerol, an endocannabinoid with important contributions to retrograde synaptic transmission.

All 10 mammalian DGK isoforms share a conserved catalytic domain and two protein kinase $\mathrm{C}$ conserved type $\mathrm{I}(\mathrm{C} 1)$ domains. The exception, with three $\mathrm{C} 1$ domains, is DGK $\theta$, the lone member of the type V DGK class and the least-studied. In their detailed review, Tu-Sekine et al. cover DGK $\theta$ structure, enzymology and the latest results, which suggest its contribution to regulation of neurotransmission.

In addition to this notable role in brain, the DGK contribution to immune response control is another area of intensive research. T lymphocyte recognition of antigens presented on the antigen-presenting cell surface generates a polarized increase in membrane DG at the contact area. Sustained DG generation at the immune synapse facilitates activation of the Ras/ERK and PKC pathways, which ultimately dictate the fate of differentiated $\mathrm{T}$ cell populations. Chen et al. extensively describe the studies with DGK $\alpha$ - and $\zeta$-deficient mice that have defined the contribution of these two isoforms to differentiation of CD8 T effector and memory populations, development of regulatory $\mathrm{T}$ cells (Treg), and lineage development of invariant natural killer cells. At difference from DGK $\alpha$, expressed predominantly in T cells, DGK $\zeta$ is expressed ubiquitously in hematopoietic cells. Singh and Kambayashi's review explores the immunomodulatory function of DGK $\zeta$ in several cell types. The dual contribution of DGK $\zeta$, which can either potentiate or limit immune responses, adds new interest to its targeting. In addition to promoting defense against infection or cancer, DGK $\zeta$ inhibition might have benefits by limiting immune responses in pathological conditions such as allergy and septic shock.

Due to their negative regulatory $\mathrm{T}$ cell function, $\mathrm{DGK} \alpha$ and $\zeta$ have recently attracted interest as prospective targets, which might improve $\mathrm{T}$ cell-mediated tumor destruction. Two articles in this series examine the findings from their own and other groups that identify DGK $\alpha$ and $\zeta$ targeting potential in the fight against cancer. The contribution of DGK $\alpha$ as a cytosolic immune checkpoint is detailed reviewed in Noessner. On their review, Riese et al. consider DGK $\alpha$ and $\zeta$ contributions to limiting antitumor functions of engineered cytotoxic T cells. In both cases, pharmacological DGK $\alpha$ inhibitors induce recovery of cytotoxic functions by tumor-infiltrating cells, although the precise role of each isoform and the consequences of specific DGK $\zeta$ inhibition remain to be addressed. The continued search for new, more effective DGK inhibitors is a critical issue that is addressed by Sakane's group, who review the recent characterization of a new $\mathrm{DGK} \alpha$ inhibitor.

DGK phosphorylation of DAG to PA is the principal pathway of DAG signaling termination. Through PA generation, DGK enzymes promote local alteration of membrane composition and electrostatic charge, which leads to recruitment and activation of additional sets of proteins. Baldanzi et al. illustrate how PA generation in specific membrane subdomains affects polarized responses such as immune synapse formation or cell migration.

We are deeply indebted to all the authors who have contributed to this Research Topic and to the dedicated reviewers who helped us achieve the highest quality standards. We gratefully acknowledge the valuable support of the Frontiers team in manuscript processing.

\section{AUTHOR CONTRIBUTIONS}

IM wrote the editorial, AG and FS approved it.

Conflict of Interest Statement: The authors declare that the research was conducted in the absence of any commercial or financial relationships that could be construed as a potential conflict of interest.

Copyright (c) 2017 Merida, Graziani and Sakane. This is an open-access article distributed under the terms of the Creative Commons Attribution License (CC BY). The use, distribution or reproduction in other forums is permitted, provided the original author(s) or licensor are credited and that the original publication in this journal is cited, in accordance with accepted academic practice. No use, distribution or reproduction is permitted which does not comply with these terms. 